\title{
Urbanidade e mortalidade por cânceres selecionados em capitais brasileiras, 1980-2009
}

\section{Urbanity and mortality by selected cancers in Brazilian capitals, 1980-2009}

\author{
Marcelle da Silva Ribeiro ${ }^{1}$, Nathália Cunha de Abreu¹, Tuane Franco Farinazzo Borges, \\ Raphael Mendonça Guimarães², Camila Drumond Muzi ${ }^{3}$
}

\begin{abstract}
Resumo
O grau de urbanização de cada estado interfere no prognóstico do câncer, já que, quanto mais urbanizado o espaço, maior o acesso à saúde. O objetivo deste estudo é analisar a tendência de mortalidade por câncer de mama, próstata e colo de útero por locais selecionados, de acordo com o grau de urbanização, no período de 1980 e 2009. Para tanto, foram calculadas as taxas de mortalidade, padronizadas para a população mundial, por câncer de mama, próstata e colo de útero para os estados brasileiros selecionados por meio do grau de urbanização e suas respectivas capitais nos anos estudados. Não houve diferença quanto à evolução da taxa de mortalidade para câncer de mama e próstata entre os estados e capitais mais urbanizados e os menos urbanizados, pois em ambos foi crescente. Nos estados mais urbanizados a taxa média foi maior do que nos menos urbanizados, indicando maior número de óbitos nestas regiões, e relação inversa foi observada para o câncer do colo de útero. Observa-se que o aumento gradativo dessa taxa reforça a necessidade de ações específicas, como as previstas no Pacto pela Vida, e agrega informações peculiaridades acerca da organização da dinâmica de diagnóstico e tratamento do câncer no país.
\end{abstract}

Palavras-chave: neoplasias da mama; neoplasias da próstata; neoplasias do colo de útero; epidemiologia; estudos de séries temporais.

\begin{abstract}
The degree of urbanization in each state interferes in cancer prognosis, since the more urbanized space, greater access to health care. The objective of this study is to analyze trends in mortality from breast cancer, prostate and cervix for selected locations, according to the degree of urbanization between 1980 and 2009. Therefore, we calculated mortality rates, standardized to the world population, breast cancer, prostate and cervix for the Brazilian states selected by the degree of urbanization and their respective capitals in the years studied. There was no difference in the evolution of mortality rates for breast cancer and prostate cancer among the states and capitals more urbanized and less urbanized, because in both cases was increasing. In the more urbanized, the average rate was higher than in less urbanized, indicating greater number of deaths in these regions, and an inverse relationship was observed for cancer of the cervix. It is observed that the gradual increase in this rate increases the need for specific actions such as those contained in the Pact for Life, and adds information about the peculiarities of the dynamics of organizational diagnosis and treatment of cancer in the country.
\end{abstract}

Keywords: breast neoplasms; prostatic neoplasms; uterine cervical neoplasms; epidemiology; time series studies.

\footnotetext{
Trabalho realizado no Instituto de Estudos em Saúde Coletiva da Universidade Federal do Rio de Janeiro (UFRJ) - Rio de Janeiro (RJ), Brasil.

${ }^{1}$ Graduanda em Enfermagem pela Escola de Enfermagem Anna Nery da UFRJ - Rio de Janeiro (RJ), Brasil.

2Doutor em Saúde Coletiva; Professor Adjunto do Instituto de Estudos em Saúde Coletiva da UFRJ - Rio de Janeiro (RJ), Brasil.

${ }^{3}$ Mestre em Ciências; Enfermeira do Instituto Nacional do Câncer - Rio de Janeiro (RJ), Brasil.

Endereço para correspondência: Raphael Mendonça Guimarães - Avenida Horácio Macedo, s/n - Ilha do Fundão - Cidade Universitária -

CEP: 21941-598 - Rio de Janeiro (RJ), Brasil - E-mail: raphael@iesc.ufrj.br

Fonte de financiamento: nenhuma.

Conflito de interesse: nada a declarar.
} 


\section{INTRODUÇÃO}

O câncer é uma patologia que resulta em grande mortalidade na população brasileira - em 2009 chegou a 17.483 casos - e representa a segunda causa de mortalidade geral ${ }^{1}$.

Com sua elevada magnitude, ele se tornou uma prioridade na saúde pública, sendo necessário um olhar epidemiológico atento e qualificado no conhecimento deste agravo tendo em vista a potencialidade de ações já existentes que ajudem a minimizar seus efeitos na população ${ }^{2}$.

Essas ações estão relacionadas à prevenção primária, ao diagnóstico precoce e à qualidade do tratamento oferecido, que se modificam de acordo com a região analisada. Sendo assim, quanto mais rápido o diagnóstico e melhor o tratamento, maiores as chances de cura.

Políticas públicas nessa área vêm sendo desenvolvidas no Brasil desde meados dos anos 1980. Mais recentemente, o controle do câncer foi afirmado como prioridade na Política Nacional de Atenção Oncológica, em 2005, e no Pacto pela Saúde, em $2006^{3}$, como estratégia para tentar minimizar as taxas de mortalidade. O Ministério da Saúde (MS) incluiu dentre as prioridades na primeira versão do Pacto pela Vida ${ }^{4}$ a redução da mortalidade pelo câncer de mama e do colo de útero, tendo como objetivos para controlar esses tipos de cânceres: ampliar a oferta de mamografia visando a alcançar cobertura de $60 \%$ da população-alvo; ampliar a oferta do exame preventivo do câncer do colo do útero a fim de alcançar $80 \%$ da população-alvo; e tratar/seguir as lesões precursoras do câncer do colo do útero no nível ambulatorial ${ }^{4}$. Na elaboração do plano plurianual 2008-2011, na inclusão de novas prioridades para o Pacto, a saúde do homem tornou-se prioridade nacional, e as diretrizes desse pacto geraram a Política de Atenção Integral à Saúde do Homem, que tem como objetivos principais diminuir a comorbidade das doenças mais prevalentes no sexo masculino, entre elas o câncer de próstata ${ }^{5}$. Contudo, estas três topografias de câncer tornam-se alvo de políticas de saúde pública prioritárias.

Por meio de estudos epidemiológicos, pode-se avaliar as taxas de mortalidade causadas por este câncer em cada região brasileira e, com isso, planejar a melhor intervenção e alcançar as metas do Pacto Pela Vida, no qual estão inclusas a redução da mortalidade por câncer de colo e mama e, ainda, a saúde do homem.

Diversos fatores explicam a variabilidade observada no uso de serviços de saúde, dentre os quais é possível citar a disponibilidade de recursos, a capacidade de compra de serviços de saúde das populações locais e os componentes sociodemográficos e epidemiológicos ${ }^{6}$.

Padrões diferenciais de incidência e mortalidade por câncer são observados entre as comunidades rurais e urbanas em todo o mundo. Fatores sociais, culturais, econômicos, profissionais, ambientais e demográficos têm sido sugeridos como grandes forças motrizes dessas disparidades. O processo de desenvolvimento econômico conduz a avanços em tecnologia médica e à melhora no acesso aos cuidados de saúde, seja para doenças infecciosas, seja para agravos não transmissíveis, como o câncer ${ }^{7}$. O grau de urbanização de cada estado, portanto, interfere no prognóstico da patologia, já que quanto mais urbanizado, maior o acesso à saúde. Assim como mostram as pesquisas, há grande dificuldade em realizar o tratamento devido à frequência e à distância de sua moradia até o local para o seu desenvolvimento, já que são localizados nas principais capitais 8 .

Ante o exposto, este estudo foi criado com o objetivo de analisar a tendência de mortalidade por câncer de mama, próstata e colo de útero por locais selecionados, de acordo com o grau de urbanização, no período de 1980 a 2009.

\section{MATERIAIS E MÉTODOS}

Foi realizado um estudo descritivo, retrospectivo de série temporal, baseado em dados secundários coletados do Departamento de Informática do Sistema Único de Saúde (DATASUS) ${ }^{9}$. Após consulta às bases de dados demográficos, observou-se que os estados com maior grau de urbanização são São Paulo, Rio de Janeiro, Paraná, Goiás e Amapá, em ordem decrescente; e com menor grau de urbanização, em ordem crescente, Rondônia, Acre, Piauí, Bahia e Maranhão.

$\mathrm{Na}$ sequência foram calculadas as taxas de mortalidade, padronizadas para a população mundial, por câncer de mama, próstata e colo de útero para os estados brasileiros selecionados por meio do grau de urbanização e suas respectivas capitais no período de 1980 a 2009. Dentre os critérios adotados, escolheram-se os cinco estados mais urbanizados e os cinco menos urbanizados, de acordo com o DATASUS. Coletaram-se os dados de mortalidade para estados selecionados e suas respectivas capitais. Para eleger os óbitos, foi incluído o código CID $10^{10}$ para câncer de próstata: C61 (neoplasia maligna da próstata). Ainda foram inclusos os códigos para câncer de mama: C50.0 (neoplasia maligna do mamilo e auréola), C50.1 (neoplasia maligna da porção central da mama), C50.2 (neoplasia maligna do quadrante superior interno da mama), C50.3 (Neoplasia maligna do quadrante inferior interno da mama), C50.4 (neoplasia maligna do quadrante superior externo da mama), C50.5 (neoplasia maligna do quadrante inferior externo da mama), C50.6 (neoplasia maligna da porção axilar da mama), C50.8 (neoplasia maligna da mama com lesão invasiva), C50.9 (neoplasia maligna da mama não especificada). E para câncer de colo de útero: 
C53.0 (neoplasia maligna na endocérvice), C53.1 (neoplasia maligna na exocérvice), C53.8 (neoplasia maligna com lesão de sobreposição de colo do útero) e C53.9 (neoplasia maligna de colo do útero não especificada).

A análise de tendência constituiu-se de cálculos das retas de modelos de regressão linear. Consideraram-se no estudo com variável dependente (Y) os coeficientes de mortalidade por câncer de próstata, e como independente $(\mathrm{X})$, o grau de urbanização. $\mathrm{O}$ modelo é dado por $\mathrm{Y}=\beta \mathrm{o}+\beta \mathrm{X}$, onde $\beta$ o é o termo constante e $\beta$ é o coeficiente de efeito linear ${ }^{11}$. As equações de tendência linear e as estatísticas de ajuste de modelo (valor de $\mathrm{R}^{2}$ e o valor $\mathrm{p}$ do teste $\mathrm{F}$ de adequação do modelo) foram obtidas do programa Statistical Package for Social Sciences (SPSS), versão 19.0.

\section{RESULTADOS}

A Tabela 1 apresenta os dados relativos à tendência da mortalidade por câncer de mama em estados e capitais brasileiras selecionados de 1980 a 2009.

É possível perceber que não houve diferença quanto à evolução da taxa de mortalidade para câncer de mama entre os estados e capitais mais urbanizados e os menos urbanizados, pois em ambos foi crescente. Entretanto, em locais como Rio Branco, Salvador, São Luís, Teresina, Amapá, Macapá, Goiânia, Curitiba e Rio de Janeiro (estado e capital) apresentou-se uma tendência não significativa $(p>0,05)$.
Estes lugares são, em sua maioria, capitais que apresentaram grandes variações nas taxas de mortalidade ao longo do tempo, o que também foi mostrado pelo coeficiente de determinação, em que seu valor é menor nas capitais.

Já na Tabela 2 podem ser vistos os dados relativos à tendência da mortalidade por câncer de próstata em estados e capitais brasileiras selecionados de 1980 a 2009.

É possível perceber que nem o mais urbanizado dos estados menos urbanizados consegue obter média próxima do estado menos urbanizado do grupo dos mais urbanizados. Além disso, é notável que a média se apresente maior nas capitais do que em seus respectivos estados, assim como o coeficiente de determinação, reforçando a alta variabilidade da tendência ao longo do tempo. Contudo, quando a magnitude do problema é demonstrada, percebemos o inverso. Neste caso, a taxa $\beta$ é maior nos estados do que em suas respectivas capitais.

Entretanto, Rio de Janeiro e São Paulo são exceções nessas situações. Apresentam taxa média maior no estado que na capital e apontam desvio-padrão discrepante em relação aos demais (19,138 e 286,002, respectivamente), justificando a alta variabilidade da taxa de mortalidade, relacionada também ao aumento da média (18,314 e 66,019). Em contrapartida, indicam coeficiente $\beta$ maior na capital do que no estado.

Finalmente, a Tabela 3 contempla os dados relativos à tendência da mortalidade por câncer do colo de útero em estados e capitais brasileiras selecionadas no período já citado anteriormente.

Tabela 1. Tendência da mortalidade por câncer de mama em estados e capitais brasileiros selecionados de 1980 a 2009

\begin{tabular}{|c|c|c|c|c|c|c|c|c|c|}
\hline $\begin{array}{l}\text { Situação de } \\
\text { urbanização }\end{array}$ & & ocal & Taxa média & $\begin{array}{l}\text { Desvio } \\
\text { padrão }\end{array}$ & $\beta$ & IC95\% & $\mathbf{R}^{2}$ & Valor $\mathbf{p}$ & Tendência \\
\hline \multirow{10}{*}{$\begin{array}{l}\text { Menor grau de } \\
\text { urbanização }\end{array}$} & Estado & Acre & 3,353 & 1,817 & 0,372 & $0,001-0,158$ & 0,139 & 0,047 & Crescente \\
\hline & Capital & Rio Branco & 4,965 & 2,960 & 0,153 & $-0,082-0,189$ & 0,024 & 0,427 & Não significativo \\
\hline & Estado & Bahia & 5,708 & 1,073 & 0,854 & $0,082-0,134$ & 0,729 & $<0,001$ & Crescente \\
\hline & Capital & Salvador & 14,517 & 2,105 & $-0,305$ & $-0,168-0,018$ & 0,093 & 0,108 & Não significativo \\
\hline & Estado & Maranhão & 2,437 & 1,120 & 0,789 & $0,072-0,136$ & 0,622 & $<0,001$ & Crescente \\
\hline & Capital & São Luís & 10,356 & 2,140 & 0,174 & $-0,054-0,141$ & 0,030 & 0,367 & Não significativo \\
\hline & Estado & Piauí & 3,913 & 1,909 & 0,751 & $0,110-0,227$ & 0,564 & $<0,001$ & Crescente \\
\hline & Capital & Teresina & 9,432 & 2,449 & 0,261 & $-0,035-0,185$ & 0,068 & 0,171 & Não significativo \\
\hline & Estado & Rondônia & 4,559 & 2,241 & 0,754 & $0,130-0,267$ & 0,569 & $<0,001$ & Crescente \\
\hline & Capital & Porto Velho & 6,618 & 4,674 & 0,694 & $0,225-0,537$ & 0,482 & $<0,001$ & Crescente \\
\hline \multirow{10}{*}{$\begin{array}{l}\text { Maior grau de } \\
\text { urbanização }\end{array}$} & Estado & Amapá & 3,707 & 2,351 & 0,315 & $-0,017-0,190$ & 0,099 & 0,096 & Não significativo \\
\hline & Capital & Macapá & 4,731 & 2,612 & 0,322 & $-0,016-0,213$ & 0,103 & 0,089 & Não significativo \\
\hline & Estado & Goiás & 7,564 & 1,510 & 0,787 & $0,096-0,183$ & 0,619 & $<0,001$ & Crescente \\
\hline & Capital & Goiânia & 12,317 & 2,345 & 0,315 & $-0,016-0,190$ & 0,099 & 0,096 & Não significativo \\
\hline & Estado & Paraná & 10,704 & 1,757 & 0,886 & $0,145-0,221$ & 0,785 & $<0,001$ & Crescente \\
\hline & Capital & Curitiba & 16,924 & 2,132 & 0,124 & $-0,067-0,129$ & 0,015 & 0,520 & Não significativo \\
\hline & Estado & Rio de Janeiro & 79,391 & 337,653 & 0,024 & $-14,708-16,601$ & 0,001 & 0,902 & Não significativo \\
\hline & Capital & Rio de Janeiro & 19,648 & 1,159 & $-0,164$ & $-0,075-0,031$ & 0,027 & 0,395 & Não significativo \\
\hline & Estado & São Paulo & 14,378 & 1,140 & 0,414 & $0,007-0,104$ & 0,172 & 0,025 & Crescente \\
\hline & Capital & São Paulo & 18,172 & 1,386 & 0,407 & $0,007-0,125$ & 0,165 & 0,029 & Crescente \\
\hline
\end{tabular}


Para este estudo, foram analisadas taxas de mortalidade por câncer de colo de útero no período de 1980 a 2009 dos cinco estados brasileiros com maior grau de urbanização e suas respectivas capitais, e dos cinco estados brasileiros com menor grau de urbanização e suas respectivas capitais.

A tendência da mortalidade por câncer de colo de útero se apresenta decrescente na maioria das capitais e estados, exceto em São Luís e no Piauí, que apresentaram tendência crescente. Porém, Acre, Rio Branco, Bahia, Rondônia, Porto Velho, Paraná e Rio de Janeiro (estado e capital) tiveram valor $p>0,05$, o que retrata uma tendência não significativa que provavelmente se deu pela flutuação da taxa.

As capitais São Luís e São Paulo apresentaram coeficientes de determinação maiores do que seus respectivos estados,

Tabela 2. Tendência da mortalidade por câncer de próstata em estados e capitais brasileiras selecionadas de 1980 a 2009

\begin{tabular}{|c|c|c|c|c|c|c|c|c|c|}
\hline $\begin{array}{l}\text { Situação de } \\
\text { urbanização }\end{array}$ & & ocal & Taxa Média & $\begin{array}{l}\text { Desvio } \\
\text { padrão }\end{array}$ & $\beta$ & IC95\% & $\mathrm{R}^{2}$ & Valor $\mathrm{p}$ & Tendência \\
\hline \multirow{10}{*}{$\begin{array}{l}\text { Menor grau de } \\
\text { urbanização }\end{array}$} & Estado & Acre & 7,159 & 2,816 & 0,509 & $0,056-0,281$ & 0,259 & 0,005 & Crescente \\
\hline & Capital & Rio Branco & 10,355 & 4,127 & 0,390 & $0,013-0,365$ & 0,120 & 0,037 & Crescente \\
\hline & Estado & Bahia & 6,762 & 2,872 & 0,898 & $0,244-0,362$ & 0,806 & $<0,001$ & Crescente \\
\hline & Capital & Salvador & 19,149 & 3,609 & 0,525 & $0,080-0,365$ & 0,275 & 0,003 & Crescente \\
\hline & Estado & Maranhão & 3,567 & 2,815 & 0,780 & $0,176-0,340$ & 0,608 & $<0,001$ & Crescente \\
\hline & Capital & São Luís & 12,768 & 4,836 & 0,702 & $0,239-0,559$ & 0,493 & $<0,001$ & Crescente \\
\hline & Estado & Piauí & 6,178 & 4,369 & 0,836 & $0,318-0,540$ & 0,699 & $<0,001$ & Crescente \\
\hline & Capital & Teresina & 13,945 & 5,780 & 0,830 & $0,414-0,713$ & 0,688 & $<0,001$ & Crescente \\
\hline & Estado & Rondônia & 9,102 & 3,277 & 0,700 & $0,161-0,378$ & 0,490 & $<0,001$ & Crescente \\
\hline & Capital & Porto Velho & 13,431 & 8,448 & 0,307 & $-0,068-0,678$ & 0,094 & 0,105 & Não significativo \\
\hline \multirow{10}{*}{$\begin{array}{l}\text { Maior grau de } \\
\text { urbanização }\end{array}$} & Estado & Amapá & 9,100 & 5,038 & $-0,190$ & $-0,342-0,117$ & 0,036 & 0,324 & Não significativo \\
\hline & Capital & Macapá & 12,197 & 7,494 & $-0,347$ & $-0,631-0,021$ & 0,120 & 0,066 & Não significativo \\
\hline & Estado & Goiás & 9,727 & 2,650 & 0,859 & $0,205-0,330$ & 0,738 & $<0,001$ & Crescente \\
\hline & Capital & Goiânia & 15,184 & 4,035 & 0,516 & $0,084-0,405$ & 0,266 & 0,004 & Crescente \\
\hline & Estado & Paraná & 12,396 & 3,742 & 0,890 & $0,312-0,470$ & 0,792 & $<0,001$ & Crescente \\
\hline & Capital & Curitiba & 17,445 & 2,463 & 0,556 & $0,066-0,256$ & 0,309 & 0,002 & Crescente \\
\hline & Estado & Rio de Janeiro & 18,314 & 19,138 & 0,116 & $-0,620-1,143$ & 0,014 & 0,548 & Não significativo \\
\hline & Capital & Rio de Janeiro & 16,640 & 1,631 & 0,625 & $0,061-0,179$ & 0,390 & $<0,001$ & Crescente \\
\hline & Estado & São Paulo & 66,019 & 286,002 & 0,188 & $-6,727-19,329$ & 0,035 & 0,830 & Não significativo \\
\hline & Capital & São Paulo & 15,742 & 2,611 & 0,689 & $0,124-0,299$ & 0,475 & $<0,001$ & Crescente \\
\hline
\end{tabular}

Tabela 3. Tendência da mortalidade por câncer do colo de útero em estados e capitais brasileiros selecionados de 1980 a 2009

\begin{tabular}{|c|c|c|c|c|c|c|c|c|c|}
\hline $\begin{array}{l}\text { Situação de } \\
\text { urbanização }\end{array}$ & \multicolumn{2}{|c|}{ Local } & \multirow{2}{*}{$\begin{array}{c}\text { Taxa Média } \\
7,3607\end{array}$} & \multirow{2}{*}{$\begin{array}{c}\text { Desvio } \\
\text { padrão } \\
3,58055\end{array}$} & \multirow{2}{*}{$\begin{array}{c}\beta \\
-0,375\end{array}$} & \multirow{2}{*}{$\begin{array}{c}\text { IC95\% } \\
-0,312--0,004\end{array}$} & \multirow{2}{*}{$\begin{array}{c}\mathbf{R}^{2} \\
0,141\end{array}$} & \multirow{2}{*}{$\begin{array}{c}\text { Valor } \mathbf{p} \\
0,312\end{array}$} & \multirow{2}{*}{$\begin{array}{c}\text { Tendência } \\
\text { Não significativo }\end{array}$} \\
\hline \multirow{10}{*}{$\begin{array}{l}\text { Menor grau de } \\
\text { urbanização }\end{array}$} & Estado & Acre & & & & & & & \\
\hline & Capital & Rio Branco & 9,1624 & 4,56869 & $-0,219$ & $-0,324--0,089$ & 0,048 & 0,254 & Não significativo \\
\hline & Estado & Bahia & 3,9759 & 0,43028 & 0,283 & $-0,005-0,033$ & 0,080 & 0,137 & Não significativo \\
\hline & Capital & Salvador & 8,0079 & 1,92773 & $-0,862$ & $-0,240--0,150$ & 0,743 & $<0,001$ & Decrescente \\
\hline & Estado & Maranhão & 5,3462 & 2,13856 & $-0,783$ & $0,135-0,283$ & 0,613 & $<0,001$ & Decrescente \\
\hline & Capital & São Luís & 18,7590 & 4,31660 & 0,749 & $-0,512--0,245$ & 0,557 & $<0,001$ & Crescente \\
\hline & Estado & Piauí & 5,0859 & 1,69558 & 0,488 & $0,029-0,166$ & 0,238 & 0,007 & Crescente \\
\hline & Capital & Teresina & 13,3076 & 5,62646 & $-0,661$ & $-0,632--0,241$ & 0,438 & $<0,001$ & Decrescente \\
\hline & Estado & Rondônia & 5,6993 & 2,37346 & 0,053 & $-0,095-0,125$ & 0,034 & 0,783 & Não significativo \\
\hline & Capital & Porto Velho & 12,7924 & 6,29237 & 0,059 & $-0,248-0,335$ & 0,003 & 0,763 & Não significativo \\
\hline \multirow{10}{*}{$\begin{array}{l}\text { Maior grau de } \\
\text { urbanização }\end{array}$} & Estado & Amapá & 14,4714 & 5,73335 & $-0,496$ & $-0,565--0,103$ & 0,246 & 0,006 & Decrescente \\
\hline & Capital & Macapá & 18,5286 & 9,15590 & $-0,550$ & $-0,946--0,237$ & 0,302 & 0,002 & Decrescente \\
\hline & Estado & Goiás & 6,8169 & 1,27069 & $-0,669$ & $-0144--0,056$ & 0,447 & $<0,001$ & Decrescente \\
\hline & Capital & Goiânia & 10,4586 & 4,01315 & $-0,805$ & $-0,490--0,269$ & 0,635 & $<0,001$ & Decrescente \\
\hline & Estado & Paraná & 5,8459 & 0,78738 & 0,234 & $-0,014-0,057$ & 0,055 & 0,223 & Não significativo \\
\hline & Capital & Curitiba & 7,9407 & 1,87169 & $-0,637$ & $-0,207--0,073$ & 0,405 & $<0,001$ & Decrescente \\
\hline & Estado & Rio de Janeiro & 6,9248 & 9,35718 & $-0,183$ & $-0,628-0,225$ & 0,034 & 0,341 & Não significativo \\
\hline & Capital & Rio de Janeiro & 4,8779 & 0,43504 & 0,137 & $-0,013-0,027$ & 0,019 & 0,478 & Não significativo \\
\hline & Estado & São Paulo & 4,7517 & 0,63596 & $-0,856$ & $-0,079--0,049$ & 0,733 & $<0,001$ & Decrescente \\
\hline & Capital & São Paulo & 5,0762 & 0,52010 & $-0,487$ & $-0,051--0,009$ & 0,237 & 0,007 & Decrescente \\
\hline
\end{tabular}


0,557 e 0,237 respectivamente, o que demonstra que a centralização do serviço de saúde se faz presente tanto nas capitais mais urbanizadas quanto nas menos urbanizadas.

Percebe-se que o desvio padrão nas capitais é maior do que em seus respectivos estados, tendo como exceção São Paulo, cujo índice é 0,636 no estado e 0,520 na capital. Isso ocorre pelo fato de que o estado, com alto grau de urbanização, não centraliza os serviços de saúde apenas na capital.

Para analisarmos a magnitude do problema, pode-se observar o coeficiente $\beta$, que exceto no Maranhão e São Luís, se apresentara menor nas capitais do que nos estados, o que não está relacionado com o grau de urbanização.

\section{DISCUSSÃO}

\section{Câncer de mama}

A taxa de mortalidade por câncer de mama no Brasil apresenta tendência de aumento entre 1980 e $2009^{1}$.

Observou-se que nos estados mais urbanizados a taxa média superou a dos menos urbanizados, indicando maior número de óbitos. Supõe-se que isto se deve à concentração do serviço de saúde nestes locais e maior exposição aos fatores de risco, o que explica também o resultado das capitais em geral que apresentam taxa média superior que os estados, como, por exemplo, Salvador e Macapá (14,517 e 4,731, respectivamente), e exceto o Rio de Janeiro. Nele, a taxa média do estado é maior do que a da capital; houve flutuação grande nos valores, como indicam o desvio-padrão $(337,653)$ e o coeficiente de correlação. Isso pode ser explicado pelo Rio de Janeiro representar uma grande capital, que recebe muito investimento governamental, além de desenvolver melhor as medidas preventivas.

Com relação à magnitude, observa-se por meio de $\beta$ que os estados têm os resultados maiores do que as capitais. Como o serviço está presente, em sua maioria, nas capitais, é compreensível o número ser mais alarmante nas demais cidades.

Uma das explicações para este crescimento está na maior exposição aos fatores de risco, como mostra uma pesquisa em Bhopal na qual se aponta que o uso de anticoncepcional oral, o aleitamento materno, o excesso de peso, obesidade e antecedentes familiares de câncer de mama foram associados significativamente à ocorrência de câncer de mama em análise multivariada ${ }^{12}$.

Por meio dos valores dos coeficientes de regressão, do coeficiente de determinação e dos valores p para a significância estatística da tendência linear, foi possível perceber que houve crescimento da mortalidade por câncer de mama tanto nos estados mais urbanizados quanto nos menos urbanizados, sendo que a taxa média indicou maior taxa de mortalidade nos estados e capitais mais urbanizados, apontando o Rio de Janeiro com a taxa mais elevada na relação dos mais urbanizados e Salvador na dos menos urbanizados.

Os estados e capitais mais urbanizados facilitam o acesso ao sistema de saúde, o que possibilita o diagnóstico precoce e o tratamento adequado da patologia. Em contrapartida, os menos urbanizados apresentam altos números de diagnóstico tardio.

Uma pesquisa realizada em Queensland, na Austrália, indica que as mulheres que residiam nas áreas socioeconomicamente desfavorecidas eram significativamente mais suscetíveis do que as moradoras das áreas mais favorecidas a serem diagnosticadas com câncer de mama avançado. As taxas foram muito maiores para as residentes em áreas afastadas do centro ${ }^{13}$.

O menor grau de urbanização dificulta a adesão ao tratamento, o que aumenta a taxa de mortalidade, tal como constatado em estudo realizado com mulheres que receberam indicação de radioterapia (RT) pós-mastectomia em locais urbanizados e não urbanizados. O estudo mostrou que as pacientes que não receberam RT experimentaram baixa sobrevida global (OS) e doença de sobrevida específica (DSS). Não residentes urbanos com local-regional de câncer de mama avançado são menos propensos a receber RT indicado. Esta discrepância pode ser devida ao acesso limitado RT em áreas não urbanas ${ }^{14}$.

A taxa média geral de mortalidade por câncer de mama também indica que houve mais mortes nas capitais do que nos estados, excetuando-se o Rio de Janeiro, pois é onde se concentra a maior parte dos atendimentos, por conta do serviço terciário de referência localizado no município do Rio de Janeiro. De fato, o Rio apresentou grande flutuação, demonstrada pelo desvio-padrão e coeficiente de correlação, o menor dentre os locais analisados.

Supõe-se que as mulheres de estados menos urbanizados se desloquem para os hospitais das capitais mais urbanizadas, onde há oferta de melhor atendimento. Em virtude disso, haveria aumento da taxa de mortalidade nos estados e capitais em questão.

Há de se considerar também que este aumento pode se relacionar com a maior capacidade de detecção dos hospitais de capitais mais urbanizadas, uma vez que dispõem de serviços de registro para quantificar os casos. Essa relação foi corroborada em uma pesquisa realizada na Carolina do Norte, que concluiu que o excesso de incidência nos municípios urbanos parece ser explicada pela preponderância urbana de hospitais de registro. Municípios com esses hospitais podem ter maior incidência por causa do aumento da detecção ${ }^{15}$.

\section{Câncer de próstata}

Os resultados encontrados apontam tendência de mortalidade maior em estados mais urbanizados, quando 
comparados aos menos urbanizados, embora haja aumento da taxa de mortalidade em todos os locais analisados. Além disso, é possível perceber que nem o mais urbanizado dos estados do grupo dos menos urbanizados consegue obter média próxima do estado menos urbanizado do grupo dos mais urbanizados, reforçando a relação inversamente proporcional da mortalidade com a urbanização.

Observa-se, inicialmente, que tanto nos estados e nas capitais mais urbanizados quanto nos menos urbanizados a tendência da mortalidade por câncer de próstata é crescente. No entanto, Porto Velho, Amapá, Macapá e os estados do Rio de Janeiro e de São Paulo apresentam tendência não significativa $(\mathrm{p}>0,05)$. Isso se dá provavelmente pela flutuação da taxa, que, neste caso, retrata muita variabilidade ao longo dos anos, representando uma tendência não linear nesses locais.

Percebe-se que os estados mais urbanizados apresentam taxa média muito mais alta quando comparados aos menos urbanizados, reflexo da concentração dos serviços de saúde nesses lugares.

As divergências observadas no presente estudo, com relação ao Rio de Janeiro e São Paulo, podem ser supostamente explicadas pelo fato deles serem os maiores centros urbanos do país, com maior suporte financeiro no investimento da saúde. Desta forma, acabam concentrando as referências de diagnóstico e tratamento do câncer de próstata, ampliando o número de internações pela doença e, consequentemente, influenciando no aumento da mortalidade nesses locais.

Colli et al. ${ }^{16}$ examinaram a associação entre as taxas de mortalidade de cânceres urológicos e a densidade médico-populacional em alguns condados dos Estados Unidos no período de 2003 a 2007. Nesse estudo foi possível observar uma sugestiva, porém não significativa, relação entre a densidade médico-populacional e as taxas de mortalidade por câncer de próstata, especificamente. Isso pode ser discrepante com nossos achados pelo fato de o acesso ao serviço de saúde e a densidade médico-populacional serem menores nos estados menos urbanizados, sugerindo maior tendência de mortalidade por câncer de próstata.

Dey et al. ${ }^{17}$ investigaram a variação da incidência de vários tipos de cânceres no Egito, relacionando com as diferenças geográficas, ambientais e demográficas das populações analisadas. Neste estudo, observou-se que a incidência urbana foi maior que a rural em todos os tipos analisados, inclusive o de próstata (Razão de Taxa de Incidência=4,85 e IC95\% 3,76-6,26). Isso se mostra coerente com nossos resultados, que apresentam a mortalidade maior nos estados mais urbanizados que nos menos urbanizados, ratificando a maior incidência da doença nos centros urbanos.
McLafferty e Wang ${ }^{18}$ analisaram a diferença de risco de câncer terminal (mama, colorretal, pulmão e próstata) entre as populações residentes em áreas urbanas e rurais no estado de Illinois, nos Estados Unidos. Este estudo sugere que os moradores rurais têm maior risco de diagnóstico tardio devido às numerosas barreiras para a obtenção de saúde preventiva, como dificuldade de acesso às redes de atenção primária e serviços de rastreamento. Apesar das barreiras apontadas pelos autores, nosso estudo aponta maior mortalidade nas áreas mais urbanizadas.

Li et al. ${ }^{19}$ analisaram a associação entre privação de vizinhança e mortalidade por câncer de próstata na Suécia. Esta pesquisa observa que a taxa de mortalidade para câncer de próstata ajustada por idade é 1,5 vez maior entre homens que vivem em locais desprovidos de vizinhança, comparada àqueles que moram nas vizinhanças mais populosas. Contudo, conclui-se que os homens que vivem em regiões menos populosas apresentam maior tendência de mortalidade para câncer de próstata. Estes dados se mostram adversos aos nossos achados, já que, de acordo com a Tabela 1, os estados e capitais menos urbanizados e, portanto, menos populosos, apresentam taxa média significativamente menor que os mais urbanizados.

Doorenbos et al..$^{20}$ avaliaram se os homens em áreas rurais e regionais da Austrália contam atualmente com acesso mais equitativo aos serviços de diagnóstico de câncer de próstata por meio das tendências para coleta do antígeno prostático específico (PSA), e a presença de câncer de próstata e da prostatectomia radical. Nesta avaliação, as taxas de incidência foram similares para os homens em áreas urbanas e rurais. No entanto, no último ano de coleta de dados, para os homens em áreas rurais, em comparação com áreas urbanas, as taxas de PSA (21.267/100.000 versus $24.606 / 100.000$; $\mathrm{p}<0,01)$ e prostatectomia radical (182,2/100.000 versus $239,2 / 100.000$; $\mathrm{p}<0,01)$ mantiveram-se inferiores e a mortalidade permaneceu maior $(56,9 / 100.000$ versus $45,8 / 100.000, p<0,01)$ nessas regiões, o que é coerente com os nossos achados.

Hayen et al. ${ }^{21}$ descreveram os padrões e tendências no tratamento cirúrgico dos homens com câncer de próstata em New South Wales, na Austrália. No momento do diagnóstico, residentes em áreas menos favorecidas socioeconomicamente foram significativamente menos propensos a sofrer prostatectomia radical após o ajuste para idade e fase da doença. Em contraponto, as taxas de orquietomia se apresentaram significativamente mais elevadas nessas mesmas regiões. $\mathrm{O}$ achado sugere dificuldade de acesso aos serviços de saúde enfrentada pela população residente nas áreas mais favorecidas socioeconomicamente, contribuindo diretamente para o diagnóstico tardio do câncer de próstata e também para o aumento da taxa de mortalidade. 


\section{Câncer do colo de útero}

Os resultados do presente estudo apontam tendência decrescente na mortalidade por câncer de colo de útero na maioria dos estados e capitais no período de 1980 a 2009, com maior tendência nas capitais e estados mais urbanizados, excetuando-se apenas São Luís e Piauí, que fazem parte do grupo dos menos urbanizados.

Observou-se que as capitais Salvador, São Luís, Teresina, Macapá, Goiânia, Curitiba e São Paulo apresentam taxa média maior do que a de seus respectivos estados, com destaque para Macapá e São Luís. Isto se dá devido à centralização dos serviços de saúde nas capitais, o que permite maior acesso a medidas preventivas que levam a um diagnóstico precoce, fazendo com que, assim, o tratamento seja iniciado com mais rapidez, resultando na diminuição da mortalidade por câncer de colo de útero.

No estudo de Singh ${ }^{22}$ realizado nos Estados Unidos entre 1969 e 2007, ambas as populações de mulheres brancas e negras em áreas não metropolitanas mantiveram as taxas de mortalidade por câncer do colo do útero significativamente maiores do que as metropolitanas, o que é coerente com o nosso estudo, no qual obtivemos os mesmos achados em relação às capitais e os estados brasileiros mais e menos urbanizados.

Em Salvador, foi observada tendência decrescente da taxa de mortalidade padronizada por câncer do colo do útero no período de 1980 a 2007, redução também verificada em todas as faixas etárias. Na Bahia houve discreto aumento na tendência da mortalidade por câncer de colo do útero ${ }^{23}$, o que é coerente com o presente estudo, que também apresenta uma taxa decrescente de mortalidade em Salvador.

Segundo Rodrigues e Teixeira ${ }^{24}$, nas capitais da região Sudeste são registradas as menores taxas de mortalidade por câncer de colo do útero (média de 12,0 por 100 mil entre os anos de 1996 e 2006), enquanto as maiores taxas (33,3 por 100 mil) são encontradas nas capitais da região Norte. Os resultados têm relação com o presente estudo, no qual dois estados da região Sudeste - Rio de Janeiro e São Paulo —, juntamente com suas respectivas capitais, apresentam as menores médias de mortalidade dentre os outros estados e capitais analisados, confirmando o declínio nas taxas de mortalidade da região.

Já no estudo de Gamarra et al. ${ }^{25}$, realizado no Nordeste do Brasil, antes da correção, os dados sugeriam que o risco de morrer por câncer de colo uterino era menor nos estados com piores índices socioeconômicos e maior nos com melhor nível, o oposto dos achados nesta pesquisa, que aponta maior mortalidade para os estados menos urbanizados, que são os de menor nível socioeconômico.

$\mathrm{O}$ trabalho de Müller et al. ${ }^{26}$ mostrou que as regiões do estado do Paraná com tendência de aumento na mortalidade apresentaram também as piores taxas em alguns indicadores socioeconômicos. Estes dados divergem com os achados do nosso estudo. Verificamos taxa média de mortalidade menor no Paraná do que em Curitiba, o que leva à reflexão que nesse estado o acesso ao serviço não se caracteriza mais eficaz na capital, mas sim no estado como um todo.

Parada et al. ${ }^{27}$ diz que a oferta do exame citopatológico tem crescido no Brasil, mas ainda é aquém da necessidade para a cobertura adequada da população-alvo, o que parece ser melhor nas capitais. Isto corrobora os achados do estudo, em que se observou maior mortalidade por câncer de colo de útero em estados do que em suas respectivas capitais, excetuando-se São Paulo.

No estudo de Lofters et al. ${ }^{28}$, realizado em Ontário, no Canadá, observa-se que as taxas de rastreamento para câncer cervical foram especialmente baixas entre mulheres com idade entre 50 a 69 anos, mulheres que vivem em áreas de baixa renda e as que possuíam plano de saúde de Ontário nos 10 anos anteriores - grupo composto, em grande parte, por imigrantes recentes. A discussão que estabelecemos no presente estudo é que a dificuldade no rastreamento é um fator que prejudica a redução das taxas de mortalidade.

Há ainda a reflexão de que a centralização do serviço e a dificuldade no rastreamento e tratamento são empecilhos para a redução da mortalidade por câncer de colo de útero. Estudo realizado pelo Instituto Nacional do Câncer ${ }^{29}$, entre 1980 e 2006, observou que apenas 30\% das mulheres se submetem ao exame citopatológico pelo menos três vezes na vida, o que resulta em diagnósticos já em fase avançada em $70 \%$ dos casos.

Pesquisas sobre mulheres grávidas na Polônia mostram que a situação, no que diz respeito à realização de exames de prevenção para câncer de colo, é significativamente pior no ambiente rural do que no urbano, e observam que a conscientização sobre os fatores de risco do câncer cervical é menor entre mulheres do meio rural $^{30}$. Isto reflete, portanto, na adesão diferenciada ao papanicolaou e, por conseguinte, na incidência e mortalidade de câncer de colo, o que é muito coerente com os achados no estudo atual.

Tran et al. ${ }^{31}$, em sua pesquisa realizada na República da Coreia, observou que $63 \%$ das participantes da região rural e $60 \%$ da urbana já haviam ouvido falar sobre o câncer cervical ( $\mathrm{p}>0,05)$. Além disso, $42 \%$ sabiam que este é o câncer mais comum do trato reprodutivo feminino e $55 \%$ que todas as mulheres estão em risco, mas apenas 36\% tinham conhecimento da prevenibilidade do câncer de colo de útero, o que tem relação com as regiões menos urbanizadas do nosso estudo, que ainda apontam taxa elevada de mortalidade poreste motivo. 
Finalmente, um estudo de realizado por Natunen et al. ${ }^{32}$ na Finlândia demonstrou que, em regiões urbanas, estratégias de vacinação recomendadas pela Organização Mundial da Saúde podem ser o suficiente para reduzir significativamente o papilomavírus humano (HPV), mas nas regiões rurais estratégias adicionais de vacinação, precisam ser consideradas. Esta medida se relaciona com o nosso estudo, no qual se observou que o estado de São Paulo apresenta tendência de mortalidade menor do que sua capital, o que, supostamente, se dá por medidas preventivas não centralizadas somente na cidade.

\section{CONCLUSÃO}

Foi possível constatar que a mortalidade pelas neoplasias selecionadas é mais elevada nos estados e capitais brasileiros com maior grau de urbanização. Isso se deve ao fato dos centros de diagnóstico e tratamento da doença se concentrarem nos centros urbanos, tanto no que se refere ao rastreamento, no caso do câncer do colo de útero, quanto para diagnóstico e tratamento, para o câncer de próstata e também de mama.
$\mathrm{O}$ aumento gradativo dessa taxa reforça a necessidade de ações específicas voltadas a homens e mulheres brasileiros e agrega informações peculiares acerca da organização da dinâmica de diagnóstico e tratamento do câncer no país.

Dentre algumas estratégias, se destaca o Pacto pela Vida, que surgiu em 2006 e abrange todo o território nacional, criando metas e buscando resultados. Uma das suas prioridades é a redução da mortalidade de câncer de colo de mama e útero. Já na segunda versão do Pacto, em 2009, a saúde do homem foi indicada como uma das prioridades da Saúde no Brasil.

Não foram encontradas muitas publicações sobre a associação entre a mortalidade por câncer e urbanização. Além disso, muitos dos estudos se apresentam inconsistentes em relação às tendências propostas, permitindo interpretações incompletas devido à escassez de pesquisas. E, ainda, não podem ser equiparadas às particularidades correlacionadas ao Brasil, como acesso ao serviço, perfil socioeconômico e hábitos de vida, uma vez que os países estudados estão inseridos em outros contextos sociais, políticos, econômicos e demográficos. Contudo, futuros estudos precisam ser encaminhados nesta direção.

\section{REFERÊNCIAS}

1. Brasil. Ministério da Saúde. Instituto Nacional de Câncer. Estimativa 2010: incidência de câncer no Brasil. Rio de Janeiro; 2009.

2. Lorenzato F, Singer A, Mould T, Santos LC, Maia A, Cariri L. Cervical cancer detection by hybrid capture and evaluation of local risk factors. Int J Gynecol Obstet. 2001;73(1):41-6.

3. Brasil. Ministério da Saúde. Instituto Nacional de Câncer. Programa Nacional de Controle do Câncer de Mama [Internet]. [cited 2012 Jan 11]. Available from: http://www2.inca.gov.br/wps/wcm/connect/acoes_ programas/site/home/nobrasil/programa_controle_cancer_mama/

4. Brasil. Ministério da Saúde. Pacto Pela Vida $1^{\text {a }}$ Versão [Internet]. [cited 2012 Jan 23]. Available from: http://dtr2001.saude.gov.br/ editora/produtos/livros/pdf/06_0257_M.pdf

5. Brasil. Ministério da Saúde. Secretaria de Atenção à Saúde. Departamento de Ações Programáticas Estratégicas. Política Nacional de Atenção Integral à Saúde do Homem (princípios e diretrizes) [Internet]. [cited 2011 Nov 29]. Available from: http://portal.saude.gov.br/portal/ arquivos/pdf/politica_nacional_atencao_integral.pdf

6. Zapponi ALB, Melo ECP. Distribuição da mortalidade por câncer de mama e de colo de útero segundo regiões brasileiras. Rev Enferm UERJ. 2010;18(4):628-31.

7. Vettore M, Lamarca G. O câncer de mama no Brasil: um problema de saúde pública que exige superação de barreiras físicas, sociais, econômicas e psicológicas [Internet]. [cited 2012 Jan 11]. Available from: http://cmdss2011.org/site/2011/12/ o-cancer-de-mama-no-brasil-um-problema-de-saude-publica-que-exigesuperacao-de-barreiras-fisicas-sociais-economicas-e-psicologicas/

8. Oliveira EXG de, Melo ECP, Pinheiro RS, Noronha CP, Carvalho MS Acesso à assistência oncológica: mapeamento dos fluxos origem-destino das internações e dos atendimentos ambulatoriais. O caso do câncer de mama. Cad Saúde Pública. 2011;27(2):317-26.
9. Brasil. Ministério da Saúde. Departamento de Informática do Sistema Único de Saúde [Internet]. [cited 2011 Nov 29]. Available from: http:// www2.datasus.gov.br/DATASUS/index.php

10. Organização Mundial da Saúde. CID-10: classificação estatística internacional de doenças e problemas relacionados à saúde. São Paulo: Edusp; 1996.

11. Latorre MRDO, Cardoso MRA. Análise de séries temporais em epidemiologia: uma introdução sobre os aspectos metodológicos. Rev Bras Epidemiol. 2001;4(3):145-52.

12. Lodha RS, Nandeshwar S, Pal DK, Shrivastav A, Lodha KM, Bhagat VK, Bankwar VV, Nandeshwar S, Saxena DM. Risk factors for breast cancer among women in Bhopal urban agglomerate: a case-control study. Asian Pac J Cancer Prev. 2011;12(8):2111-5.

13. Baade PD, Turrell G, Aitken JF. Geographic remoteness, area-level socioeconomic disadvantage and advanced breast cancer: a cross-sectional, multilevel study. J Epidemiol Community Health. 2011;65(11):1037-43.

14. Martinez SR, Tseng WH, Shah DR, Canter RJ, Bold RJ. Urban and nonurban disparities in the use of post-mastectomy radiation for breast cancer. Med Oncol. 2012;29(3):1523-8.

15. Hall SA, Kaufman JS, Millikan RC, Ricketts TC, Herman D, Savitz DA. Urbanization and breast cancer incidence in North Carolina, 1995-1999. Ann Epidemiol. 2005;15(10):796-803.

16. Colli J, Sartor O, Thomas R, Lee BR. Does urological cancer mortality increase with low population density of physicians? J Urol.2011;186(6):2342-6.

17. Dey S, Zhang Z, Hablas A, Seifeldein IA, Ramadan M, El-Hamzawy H, Soliman AS. Geographic patterns of cancer in the population-based registry of Egypt: possible links to environmental exposures. Cancer Epidemiol. 2011;35(3):254-64 
18. McLafferty S, Wang F. Rural reversal? Rural-urban disparities in latestage cancer risk in Illinois. Cancer. 2009;115(12):2755-64.

19. Li X, Sundquist K, Sundquist J. Neighborhood deprivation and prostate cancer mortality: a multilevel analysis from Sweden. Prostate Cancer Prostatic Dis. 2012;15(2):128-34.

20. Doorenbos AZ, Jacobsen C, Corpuz R, Forquera R, Bunchwald D. A randomized controlled calendar mail-out to increase cancer screening among urban American Indian and Alaska Native patients. J Cancer Educ. 2011;26(3):549-54.

21. Hayen A, Smith DP, Patel MI, O'Connell DL. Patterns of surgical care for prostate cancer in NSW, 1993-2002: rural/urban and socio-economic variation. Aust N Z J Public Health. 2008;32(5):417-20.

22. Singh GK. Rural-urban trends and patterns in cervical cancer mortality, incidence, stage, and survival in the United States, 1950-2008. J Community Health. 2012;37(1):217-23.

23. Junior ACS, Rêgo MAV. Tendência da mortalidade por câncer de colo do útero em Salvador e no estado da Bahia, Brasil, de 1980 a 2007. Revista Baiana de Saúde Pública; 35(3):722-33.

24. Rodrigues AD, Teixeira MTB. Mortalidade por câncer de mama e câncer de colo do útero em município de porte médio da Região Sudeste do Brasil, 1980-2006. Cad Saúde Pública. 2011;27(2):241-8.

25. Gamarra CJ, Valente JG, Silva GA. Magnitude da mortalidade por câncer do colo do útero na Região Nordeste do Brasil e fatores socioeconômicos. Rev Panam Salud Publica. 2010;28(2):100-6.

26. Müller EV, Biazevic MGH, Antunes JLF, Crosato EM. Tendência e diferenciais socioeconômicos da mortalidade por câncer de colo de útero no Estado do Paraná (Brasil), 1980-2000. Ciênc Saúde Coletiva. 2011:16(5):2495-500.

27. Parada R, Assis M, Silva RCF, Abreu MF, Silva MAF, Dias MBK, Tomazelli JG. A Política Nacional de Atenção Oncológica e o papel da atenção básica na prevenção e controle do câncer. Rev APS. 2008:11(2):199-206.

28. Lofters AK, Moineddin R, Hwang SW, Glazier RH. Low rates of cervical cancer screening among urban immigrants: a population-based study in Ontario, Canada. Med Care. 2010;48(7):611-8.

29. Instituto Nacional do Câncer. Programa Nacional de Controle do Câncer do Colo do Útero e de Mama - Viva Mulher. Prevenção e Detecção [Internet]. [cited 2010 Nov. 26]. Available from: http://www2.inca.gov. $\mathrm{br} / \mathrm{wps} / \mathrm{wcm} /$ connect/acoes_programas/site/home/nobrasil/programa_ nacional_controle_cancer_colo_utero

30. Bojar I, Bilinski P, Boyle P, Zatonski W, Marcinkowski JT, Wojtyla A. Prevention of female reproductive system cancer among rural and urban Polish pregnant women. Ann Agric Environ Med. 2011;18(1):183-8.

31. Tran NT, Choe SI, Taylor R, Ko WS, Pyo HS, So HC. Knowledge, attitude and practice (KAP) concerning cervical cancer and screening among rural and urban women in six provinces of the Democratic People's Republic of Korea. Asian Pac J Cancer Prev. 2011;12(11):3029-33.

32. Natunen K, Lehtinen J, Namujju P, Sellors J, Lehtinen M. Aspects of prophylactic vaccination against cervical cancer and other human papillomavirus-related cancers in developing countries. Infect Dis Obstet Gynecol. 2011. Epub 2011 Jul 19.

Recebido em: 08/05/2012 Aprovado em: 19/09/2012 